\title{
Irreversibility of the two-dimensional enstrophy cascade
}

\author{
E. Piretto, ${ }^{1}$ S. Musacchio, ${ }^{2}$ F. De Lillo, ${ }^{1}$ and G. Boffetta ${ }^{1}$ \\ ${ }^{1}$ Department of Physics and INFN, Università di Torino, 1 Via P. Giuria, 10125 Torino, Italy \\ ${ }^{2}$ Laboratoire Jean-Alexandre Dieudonné, Université de Nice Sophia Antipolis, CNRS, UMR No. 7351, 06100 Nice, France
}

(Received 19 July 2016; published 22 November 2016)

\begin{abstract}
We study the time irreversibility of the direct cascade in two-dimensional turbulence by looking at the time derivative of the square vorticity along Lagrangian trajectories, a quantity called metenstrophy. By means of extensive direct numerical simulations we measure the time irreversibility from the asymmetry of the probability density function of the metenstrophy and we find that it increases with the Reynolds number of the cascade, similarly to what is found in three-dimensional turbulence. A detailed analysis of the different contributions to the enstrophy budget reveals a remarkable difference with respect to what is observed for the energy cascade, in particular the role of the statistics of the forcing to determine the degree of irreversibility.
\end{abstract}

DOI: 10.1103/PhysRevE.94.053116

\section{INTRODUCTION}

Although the time reversibility of the Navier-Stokes equations is broken by the viscous forces, it is not restored in the limit of vanishing viscosity. Indeed, this limit of fully developed turbulence is characterized by an irreversible flux of energy from the large scales to the small scales where it is dissipated. The irreversibility of the energy flux in threedimensional (3D) turbulence is responsible for the asymmetry of the two-point statistical observables, either in a fixed Eulerian reference frame as in the case of velocity structure functions that display a negative third moment [1] or for the Lagrangian evolution of pairs of trajectories [2].

Recently, it has been shown on the basis of laboratory experiments and numerical simulations how irreversibility in turbulence manifests itself at the level of a single-point observable [3]. By looking at the evolution of the (kinetic) energy along a fluid trajectory, it has been shown that the particle acquires energy on a long time scale and loses it on a short time scale. This reflects the fact that energy is injected in the flow by an external forcing at large slow scales and is dissipated by viscosity at small fast scales [4]. As a consequence, although in stationary conditions the mean temporal increment of energy vanishes, the full statistics is not time reversible and odd moments of energy increments are different from zero [3]. Time irreversibility can be quantified by looking at the statistics of the power along a trajectory $p(t)=d / d t\left[v^{2}(t) / 2\right]$ and it has been shown that the third moment $\left\langle p^{3}\right\rangle$ is negative and increases with the control parameter, the Reynolds number, of the flow. Similar results have been found for the inverse cascade of energy in two-dimensional turbulence, which is characterized by the same scaling law of 3D turbulence [5] and in compressible turbulence [6]. Despite the fact that in two dimensions the energy flows towards the large scales (instead of the small scales), time irreversibility manifests in two-dimensional turbulence as in three dimensions, with a negative skewness of the power computed along a Lagrangian trajectory [3].

In this paper we study the time irreversibility of the direct cascade in two-dimensional turbulence, characterized by an enstrophy (mean square vorticity) flow towards small scales, by studying the statistics of what we call metenstrophy, i.e., the time derivative of the enstrophy along a trajectory. The main motivation for this work is that the direct cascade is characterized by a single characteristic time [5] and therefore spatial separation (between injection and dissipation scales) does not correspond in a simple way to time-scale separation. This make the enstrophy cascade completely different from the energy cascades (direct and inverse) studied in [3]. On the basis of direct numerical simulations at different Reynolds numbers, we find that also in this case single-point statistics breaks time-reversal symmetry and that the degree of irreversibility grows with the Reynolds numbers of the flow. Apart from this similarity, the picture that emerges from the enstrophy cascade is very different from what is observed in the energy cascade, as here the terms that contribute to the enstrophy balance are all local and the pressure gradient, which is responsible for the transfer of energy from slow to fast particles in three dimensions [7], is absent.

The remaining part of this paper is organized as follows. In Sec. II we provide a brief survey of two-dimensional turbulence, introducing the equations and the main quantities that will be studied. In Sec. III we report the results of our numerical simulations. Section IV is devoted to the discussion of our findings.

\section{THEORETICAL BACKGROUND}

We consider the two-dimensional incompressible NavierStokes equation for the vorticity field $\omega=\partial_{x} u_{y}-\partial_{y} u_{x}$ in a double periodic square box of dimension $L \times L$,

$$
\partial_{t} \omega+\boldsymbol{u} \cdot \nabla \omega=v \nabla^{2} \omega-\alpha \omega+f
$$

where $v$ is the kinematic viscosity, $\alpha$ is the friction coefficient, and $f(\boldsymbol{x}, t)$ is an external forcing needed to sustain a stationary state. In the inviscid $(v=\alpha=0)$ unforced $(f=0)$ limit (1) has two conserved quantities: kinetic energy $E=(1 / 2)\left\langle u^{2}\right\rangle$ and enstrophy $Z=(1 / 2)\left\langle\omega^{2}\right\rangle$. Here $\langle\cdots\rangle$ represents an average over the $L^{2}$ domain. The forcing term $f$ injects energy and enstrophy at a characteristic scale from which they are transported towards large and small scales, respectively, generating the double cascade predicted by Kraichnan 50 years ago $[5,8]$. The inverse cascade of energy is characterized by a Kolmogorov-like spectrum with close-to-Gaussian statistics [9] and its time-reversal properties have been the subject of previous works $[3,7]$. In the direct cascade enstrophy is transferred at a rate $\eta$ from the forcing scales $\ell_{f}$ down to the small dissipative scales $\ell_{v} \sim v^{1 / 2} / \eta^{1 / 6}$ generating a power-law 
TABLE I. Parameters of the simulations with deterministic forcing (set A). The amplitude of the force is $F=1$ and the forcing wave number $k_{f}=4$. The hypofriction coefficient is $\alpha=1$.

\begin{tabular}{lccccr}
\hline \hline $\operatorname{Re}$ & $v$ & $-\left\langle q_{v}\right\rangle$ & $-\left\langle q_{\alpha}\right\rangle$ & $\left\langle q_{f}\right\rangle$ & $E$ \\
\hline $7.8 \times 10^{2}$ & $10^{-3}$ & $2.48 \times 10^{-1}$ & $2.4 \times 10^{-2}$ & $2.72 \times 10^{-1}$ & $1.06 \times 10^{-1}$ \\
$1.5 \times 10^{3}$ & $5 \times 10^{-4}$ & $2.24 \times 10^{-1}$ & $2.8 \times 10^{-2}$ & $2.53 \times 10^{-1}$ & $1.30 \times 10^{-1}$ \\
$3.7 \times 10^{3}$ & $2 \times 10^{-4}$ & $2.13 \times 10^{-1}$ & $3.4 \times 10^{-2}$ & $2.47 \times 10^{-1}$ & $1.68 \times 10^{-1}$ \\
$7.3 \times 10^{3}$ & $1 \times 10^{-4}$ & $2.07 \times 10^{-1}$ & $3.7 \times 10^{-2}$ & $2.44 \times 10^{-1}$ & $1.38 \times 10^{-1}$ \\
$1.5 \times 10^{4}$ & $5 \times 10^{-5}$ & $2.05 \times 10^{-1}$ & $4.0 \times 10^{-2}$ & $2.46 \times 10^{-1}$ & 2.62 \\
\hline \hline
\end{tabular}

spectrum with an exponent close to the dimensional prediction -3 [10-17]. The ratio of these scales defines the Reynolds number as the ratio of the forcing and dissipative scales of the cascade as $\operatorname{Re}=\eta^{1 / 3} \ell_{f}^{2} / \nu=\left(\ell_{f} / \ell_{v}\right)^{2}$.

In the presence of forcing and dissipation, the time derivative of the local square vorticity along a trajectory, which will be called metenstrophy $q$, is given, from (1), by

$$
q \equiv \frac{D}{D t} \frac{1}{2} \omega^{2}=q_{v}+q_{\alpha}+q_{f}
$$

where with obvious notation we have introduced $q_{v}=\omega \nu \nabla^{2} \omega$, $q_{\alpha}=-\alpha \omega^{2}$, and $q_{f}=\omega f$. In stationary conditions we have $\langle q\rangle=0$ and the enstrophy balance reads $\left\langle q_{f}\right\rangle=-\left\langle q_{v}\right\rangle-\left\langle q_{\alpha}\right\rangle$, where the viscous dissipation is equal to the enstrophy flux $-\left\langle q_{\nu}\right\rangle=\eta$, while the large scale enstrophy dissipation $\left\langle q_{\alpha}\right\rangle$ is negligible for large Reynolds numbers [9].

We observe that in the decomposition (2) all terms are local, involving products of the vorticity field and its derivatives. This is the main difference with respect to the balance for the energy cascade in which a nonlocal term, given by the pressure gradient, is present. Although the pressure forces on average do not contribute to the kinetic energy balance, in three dimensions they are responsible for the redistribution of energy from slow to fast particles and for the asymmetry of the probability density function (PDF) of energy power. The absence of the analogous pressure forces in (2) suggests that the statistics of metenstrophy in the $2 \mathrm{D}$ direct cascade will be very different from the statistics of power in three dimensions.

\section{NUMERICAL RESULTS}

We have integrated the Navier-Stokes equations (1) on a double periodic square domain of size $L=2 \pi$ at resolution $N^{2}=1024^{2}$, by means of a standard fully dealiased pseudospectral code, with a fourth-order Runge-Kutta scheme with implicit integration of the linear dissipative terms. In order to avoid large-scale contamination of the enstrophy cascade [18], the linear friction term $-\alpha \omega$ has been numerically replaced by a hypofriction term $-\alpha \nabla^{-4} \omega$, which confines the dissipation to the largest scales.

Because the variations of the enstrophy along a Lagrangian trajectory are only due to the contributions of the forcing and dissipation, we have performed two sets of simulations aimed at investigating separately the effects of the viscous dissipation and of the external forcing on the metenstrophy statistics. In the following, all the results are nondimensionalized with the enstrophy flux $\eta$ and with the characteristic time of the flow defined as $\tau_{\omega}=\eta^{-1 / 3}$.

\section{A. Dependence on Reynolds number}

We performed a first set of simulation (set A) to investigate the dependence of the statistics on the Reynolds number by gradually reducing the viscosity and keeping the forcing fixed. In these simulations we use a deterministic time-independent forcing $f(x, y)=F \sin \left(k_{f} x\right) \sin \left(k_{f} y\right)$. The forcing scale is defined as $\ell_{f}=2 \pi / k_{f} \sqrt{2}$ and the parameters of the simulations are reported in Table I. The statistics is computed over 2000 independent vorticity fields, sampled every $1.2 \tau_{\omega}$.

In Fig. 1 we show the PDFs of the metenstrophy $q$ for the simulations of set A at different Reynolds numbers. Even if the mean value of $q$ vanishes, because of the statistical time stationarity, the full statistics reveals a noticeable violation of the time symmetry. In particular, at increasing Re we observe the development of a large left tail in the PDF and the third moment of $q$ become negative.

Figure 2 shows the second moment $\left\langle q^{2}\right\rangle$ and the third moment $\left\langle q^{3}\right\rangle$ of the distribution of metenstrophy as a function of the Reynolds number. Both moments are found to grow monotonically with $\mathrm{Re}$, in particular the dimensionless quantity $-\left\langle q^{3}\right\rangle / \eta^{3}$, which provides a suitable measure of irreversibility, similarly to what is observed for the statistics of power in 3D turbulence [3]. At variance with the 3D case, here we are unable to find a clear power-law scaling for the two moments, but this could still be due to the finite Reynolds effect.

The inset of Fig. 2 shows that the skewness $S=\left\langle q^{3}\right\rangle /$ $\left\langle q^{2}\right\rangle^{3 / 2}$ also increases in magnitude with the Reynolds number of the flow and suggests a possible saturation to a constant

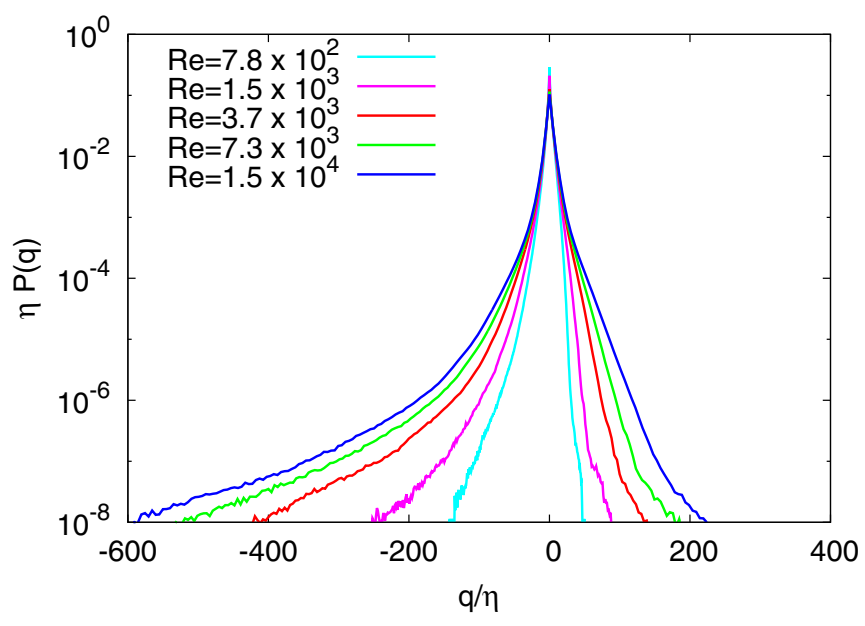

FIG. 1. Probability density functions of metenstrophy $q$ at increasing Re (from the inner to the outer PDF). 


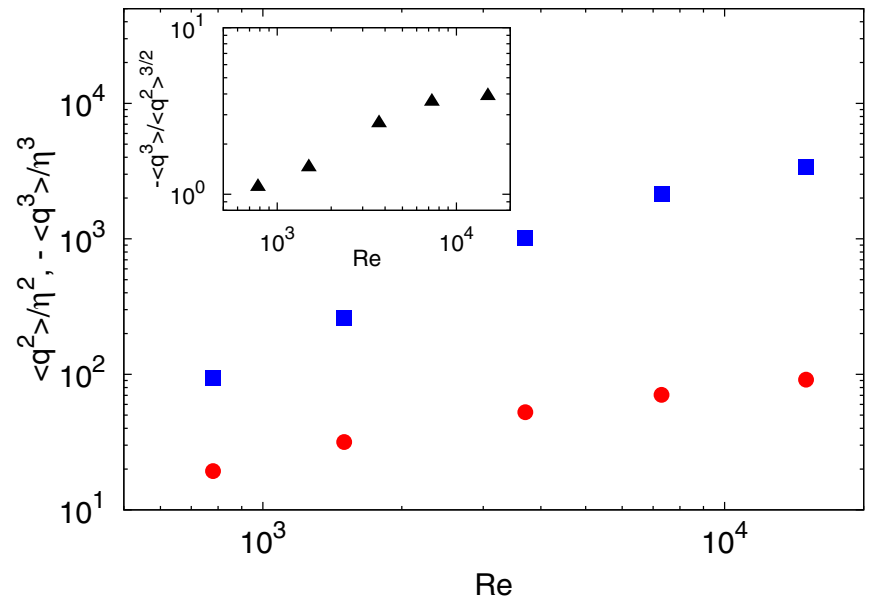

FIG. 2. Second (red circles) and third (blue squares) moments of the PDFs of metenstrophy $q$ as a function of Re. The inset shows the skewness of metenstrophy as a function of Re.

value for large values of Re. We remark that in the case of the energy cascade in both $2 \mathrm{D}$ and $3 \mathrm{D}$ turbulence, the skewness of the PDF of the power is found to be nearly independent of $\operatorname{Re}[3]$.

In order to understand which physical process is responsible for the breaking of the time asymmetry, we have analyzed the different contributions (2) to the metenstrophy, due to the forcing $q_{f}$, the viscous dissipation $q_{\nu}$, and the large-scale friction $q_{\alpha}$. In Fig. 3 we compare the PDF of $q$ for the run at $\operatorname{Re}=1.5 \times 10^{4}$ with the PDFs of $q_{f}, q_{\nu}$, and $q_{\alpha}$. We find that the large left (negative) tail of the PDF of $q$ is dominated by local events of intense viscous dissipation, which can be 600 times more intense than their mean. Conversely, the statistics of the forcing contributions $q_{f}$ is more symmetric and it prevails in the right (positive) tail of $P(q)$. As expected, the contribution of the large-scale friction to the statistics of $q$ is negligible.

It is remarkable that we observe a signature of the presence of strong dissipative events (associated with the left tail in Fig. 3) also in the statistical convergence of the moments

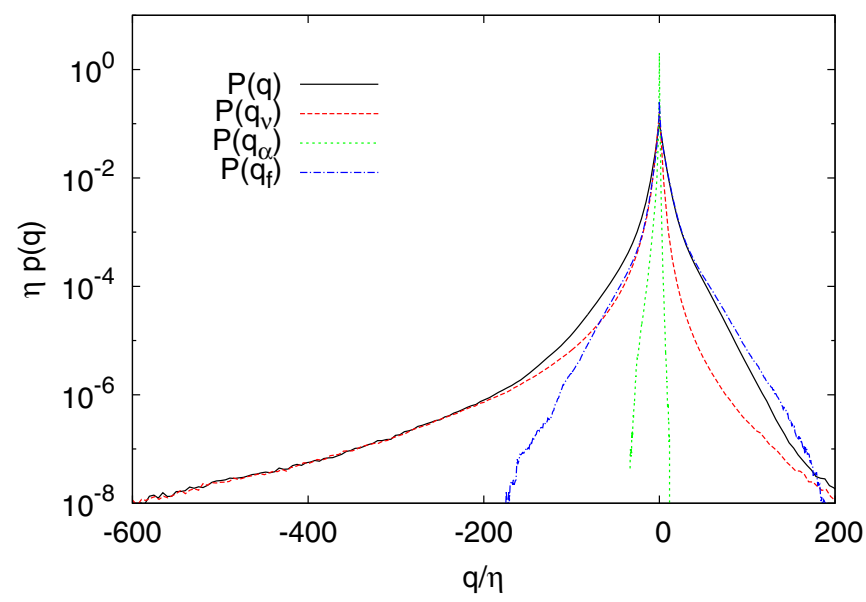

FIG. 3. The PDF of the metenstrophy $q$ for the case $\mathrm{Re}=1.5 \times$ $10^{4}$ (back solid line) and of the different contributions: viscosity $q_{v}$ (red dashed line), friction $q_{\alpha}$ (green dotted line), and forcing $q_{f}$ (blue dash-dotted line).
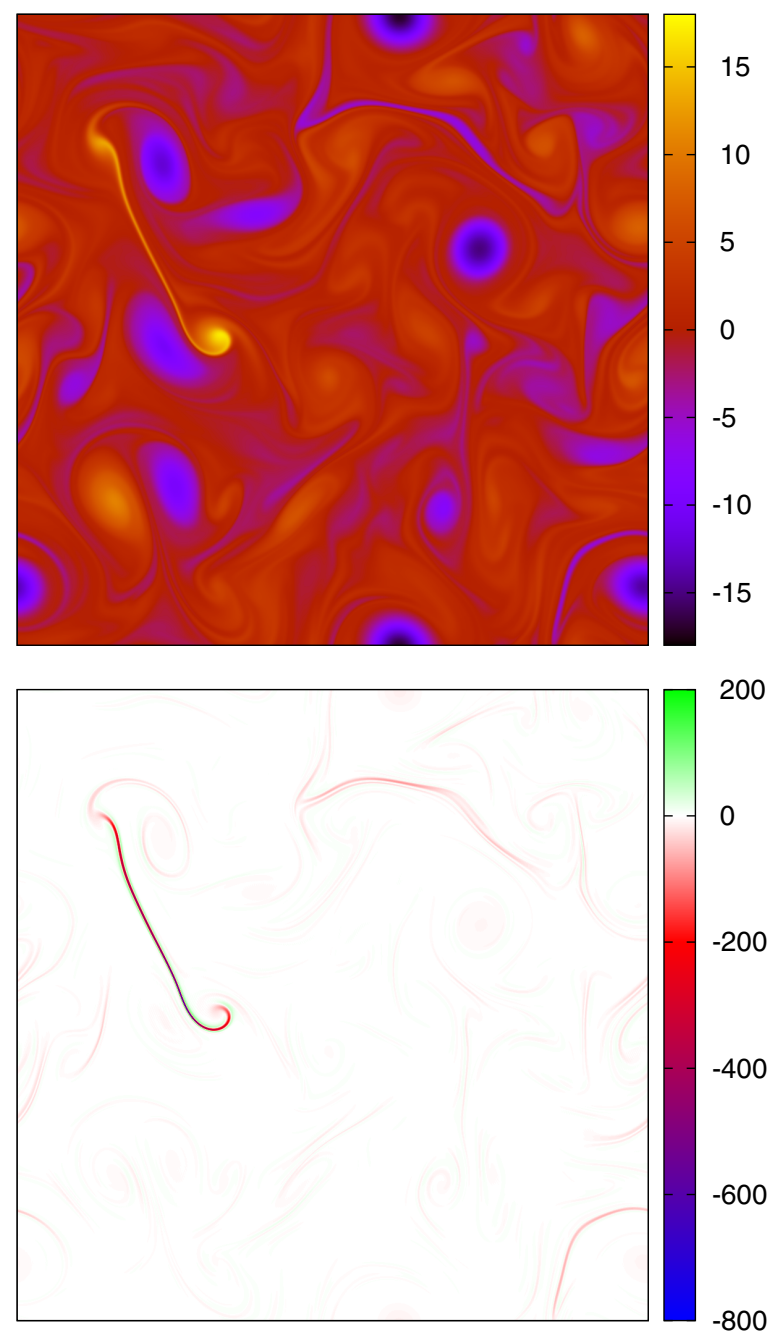

FIG. 4. Snapshots of the vorticity field $\omega$ (top) and viscous enstrophy dissipation rate $q_{v}$ (bottom) at the same time for the simulation at $\operatorname{Re}=3.7 \times 10^{3}$.

of $q$, which displays abrupt changes of the averages during the time evolution of the system. A visual inspection of the vorticity field during one of these events reveals the presence of extremely intense and tiny filaments of vorticity (see Fig. 4). These vorticity filaments, which are generated by the chaotic stretching of the direct enstrophy cascade [19], causes localized events of strong viscous dissipation, which are clearly visible in the corresponding field $q_{v}$ shown in Fig. 4.

In summary, the results of this set of simulations reveal a significant breaking of the time symmetry in the statistics of the metenstrophy. The irreversibility increases with Reynolds number and it is intrinsically related to the chaotic-stretching nature of the direct enstrophy cascade, which produces tiny and intense filaments of vorticity localized in both space and time.

\section{B. Dependence on the forcing correlation time}

Considering that the intense dissipative events must be balanced on average by the forcing, it is natural to suppose that the statistics of the metenstrophy cannot be universal with respect to the forcing itself. We addressed this issue 
TABLE II. Parameters of the simulations with Ornstein-Uhlenbeck forcing (set B). The wave-number forcing shell is $5 \leqslant k \leqslant 6$. The viscosity is $v=5 \times 10^{-5}$ and the friction coefficient is $\alpha=1$.

\begin{tabular}{|c|c|c|c|c|c|c|c|c|}
\hline $\mathrm{Re}$ & $\tau_{f} \eta^{1 / 3}$ & $\tau_{f}$ & $F$ & $-\left\langle q_{v}\right\rangle$ & $-\left\langle q_{\alpha}\right\rangle$ & $\left\langle q_{f}\right\rangle$ & $E$ & $Z$ \\
\hline $1.60 \times 10^{4}$ & 0.15 & 0.25 & $2.5 \times 10^{-1}$ & $2.08 \times 10^{-1}$ & $3.8 \times 10^{-2}$ & $2.50 \times 10^{-1}$ & $1.60 \times 10^{-1}$ & 3.52 \\
\hline $1.55 \times 10^{4}$ & 0.29 & 0.50 & $5.8 \times 10^{-2}$ & $1.89 \times 10^{-1}$ & $3.5 \times 10^{-2}$ & $2.25 \times 10^{-1}$ & $1.55 \times 10^{-1}$ & 3.45 \\
\hline $1.63 \times 10^{4}$ & 0.60 & 1.00 & $2.0 \times 10^{-2}$ & $2.18 \times 10^{-1}$ & $4.1 \times 10^{-2}$ & $2.60 \times 10^{-1}$ & $1.80 \times 10^{-1}$ & 4.05 \\
\hline $1.55 \times 10^{4}$ & 1.15 & 2.00 & $5.7 \times 10^{-3}$ & $1.87 \times 10^{-1}$ & $3.7 \times 10^{-2}$ & $2.25 \times 10^{-1}$ & $1.68 \times 10^{-1}$ & 3.90 \\
\hline $1.57 \times 10^{4}$ & 2.91 & 5.00 & $1.8 \times 10^{-3}$ & $1.96 \times 10^{-1}$ & $4.0 \times 10^{-2}$ & $2.37 \times 10^{-1}$ & $1.92 \times 10^{-1}$ & 3.53 \\
\hline
\end{tabular}

with a second set of simulations (set B), in which we keep the viscous dissipation fixed and we have changed the time correlation $\tau_{f}$ of the external forcing. For this purpose, we forced all the wave numbers in the shell $k \in\left[k_{f 1}, k_{f 2}\right]$ with independent stochastic Ornstein-Uhlenbeck processes $d f_{t, k}=$ $-\left(1 / \tau_{f}\right) f_{t, \boldsymbol{k}} d t+\sqrt{2 F} d W_{t, \boldsymbol{k}}$, where $W_{t, \boldsymbol{k}}$ are independent Wiener processes. The amplitude $F$ of the forcing has been tuned to obtain, a posteriori, similar enstrophy fluxes in the simulations with different $\tau_{f}$. We define the mean square forcing wave number as $k_{F}^{2}=\left\langle|\boldsymbol{k}|^{2}\right\rangle_{k \in\left[k_{f 1}, k_{f 2}\right]}$ and the forcing scale as $\ell_{f}=2 \pi / k_{f}$. The parameters of this second set of simulations are reported in Table II. Also in this case, the statistics is computed over 2000 independent vorticity fields, sampled every $1.2 \tau_{\omega}$.

The PDFs of the metenstrophy computed in the simulations with different forcing are shown in Fig. 5. When the forcing has a long correlation time $\tau_{f}$ (longer than $\tau_{\omega}$ ), we observe a PDF characterized by a strong asymmetry, due to a pronounced left tail, similar to what is observed for the stationary forcing of set A. However, we find that the asymmetry diminishes as we reduce the correlation time of the forcing.

The symmetrization of the PDF is accompanied by a broadening of its tails. This is well captured by the dependence of the second and third moments on $\tau_{f}$ shown in Fig. 6. As the correlation time is reduced, we observe an increase of the second moment, which corresponds to the broadening of the PDF's tails, and a reduction of the third moment of the distributions, which indicates the reduction of the asymmetry.

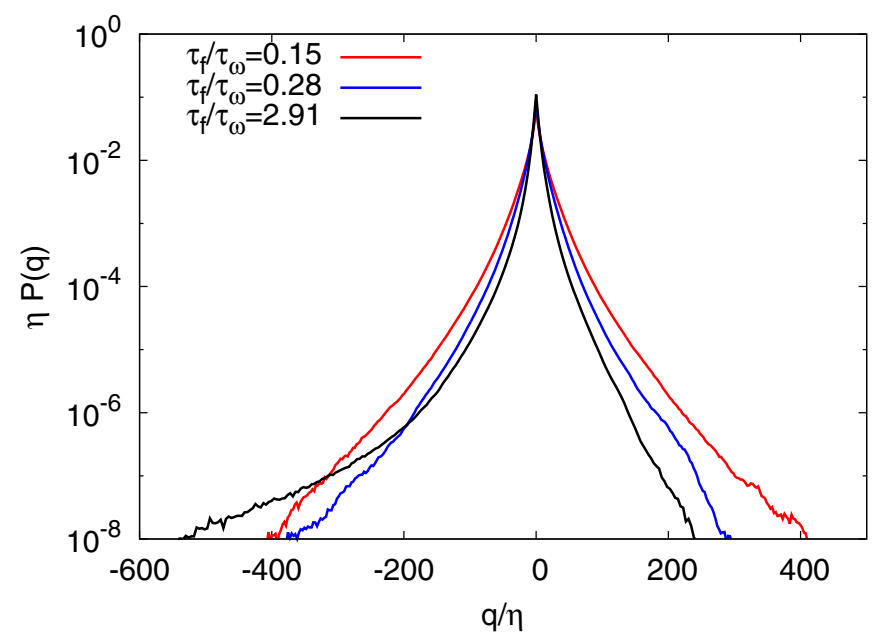

FIG. 5. The PDFs of metenstrophy $q$ for different correlation times $\tau_{f}\left(\tau_{f} / \tau_{\omega}\right.$ increases from the outer to the inner curve).
The combined growth of $\left\langle q^{2}\right\rangle$ and the decrease of $\left\langle q^{3}\right\rangle$ results in a reduction of the skewness $S=\left\langle q^{3}\right\rangle /\left\langle q^{2}\right\rangle^{3 / 2}$ at reducing the correlation time of the forcing (see Fig. 6). The analysis of the different contributions to the metenstrophy (the forcing $q_{f}$, the viscous dissipation $q_{v}$, and the friction $q_{\alpha}$ ) reveals that the fluctuations of the forcing are mainly responsible for the broadening of the tails observed in the case of short correlated forcing (see Fig. 7). The PDF of the viscous dissipation $q_{v}$ displays a clear asymmetry, but its contribution to $P(q)$ is in this case much weaker than that of the forcing.

Our findings have a simple explanation. Reducing the time correlation of the forcing causes also a reduction of the correlation between the force field and the vorticity field. The mean, positive enstrophy input (which is fixed) is therefore the result of cancellations between larger and larger positive and negative fluctuations of the input, which have no reason to be asymmetric. The broad symmetric tails of $P\left(q_{f}\right)$, which develops in the limit $\tau_{f} \rightarrow 0$, overwhelm the asymmetric contributions of $P\left(q_{v}\right)$, originated by the generation of the tiny vorticity filaments.

\section{CONCLUSION}

In this work we have investigated the statistics of the metenstrophy $q$, the time derivative of the enstrophy along a Lagrangian trajectory, in two-dimensional turbulent flows in the regime of the direct enstrophy cascade sustained by

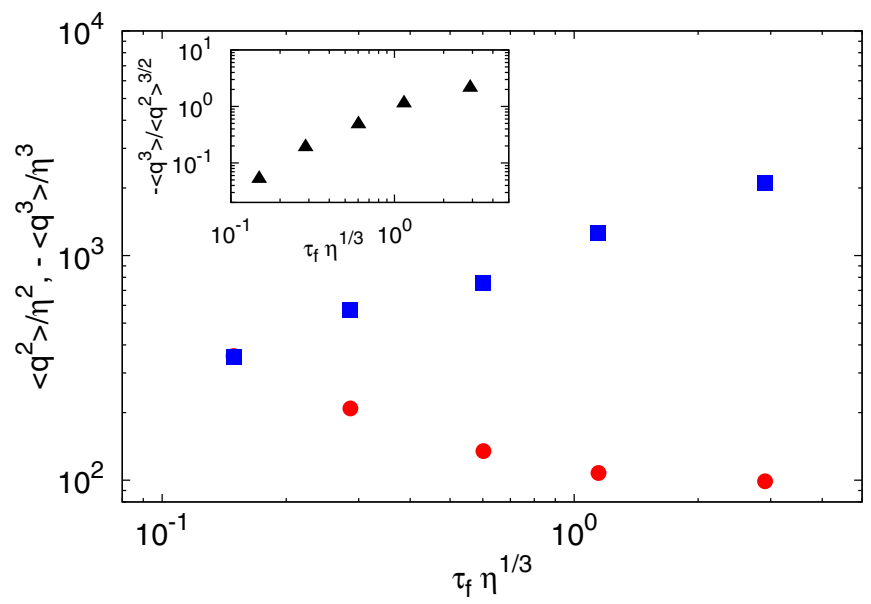

FIG. 6. Second (red circles) and third (blue squares) moments of the PDF of metenstrophy $q$ as a function of the forcing correlation time $\tau_{f}$. The inset shows the skewness of metenstrophy as a function of $\tau_{f}$. 


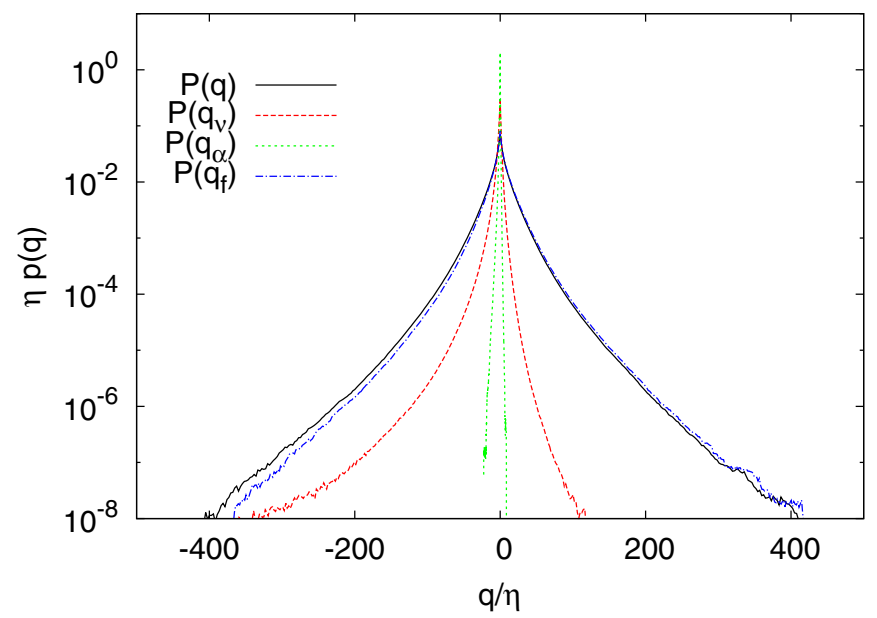

FIG. 7. The PDF of the metenstrophy $q$ for the case $\tau_{f} \eta^{1 / 3}=0.15$ (back solid line) and of the different contributions: viscosity $q_{v}$ (red dashed line), friction $q_{\alpha}$ (green dotted line), and forcing $q_{f}$ (blue dash-dotted line).

deterministic and stochastic forcing. The main result of our work is that the statistics of $q$ is characterized by a strong violation of the time symmetry. The irreversibility increases with the Reynolds number and it is closely related to the mechanism of the direct enstrophy cascade, which generates tiny filaments of vorticity by means of chaotic stretching. At the viscous scales, these filaments cause intense events of enstrophy dissipation, therefore giving strong contributions to the left negative tail of the PDF of $q$.

As the result of balance between forcing and dissipation, the statistics of the metenstrophy depends on the forcing mechanisms. In particular, we have shown that the irreversibility is reduced in the case of stochastic forcing with short correlation time, whose broad and symmetric fluctuations overcome the asymmetric contribution given by the viscous dissipation.

The mechanism that causes the symmetry breaking is essentially the chaotic stretching of the flow. This suggests that our results can be extended also to other systems in similar conditions, in particular to the statistics of a scalar field transported by the turbulence of a chaotic flow. More generally, the study of single-point irreversibility in different turbulence models will allow one to build a general picture of possible universal features of how time symmetry breaks in far-from-equilibrium systems.

Two-dimensional turbulence is characterized by the presence of two simultaneous cascades of energy and enstrophy. The irreversibility of the two cascades can be measured by the asymmetry of the statistics of the power and the metenstrophy, respectively. The third moments of the power $\left\langle p^{3}\right\rangle$ and of the metenstrophy $\left\langle q^{3}\right\rangle$ are both negative, but the mechanisms for the symmetry breaking in the two cascades are different. In the case of the inverse energy cascade it is mainly due to inertial contributions of the pressure gradients [3]. Conversely, in the case of the direct enstrophy cascade it is caused by extreme dissipative events. Moreover, the irreversibility measured by $\left\langle q^{3}\right\rangle$ grows with the extension of the inertial range of the direct enstrophy cascade, at variance with $\left\langle p^{3}\right\rangle$, which grows with the extension of the inertial range of the inverse energy cascade [3]. The turbulent flows investigated here are sustained by a large-scale forcing and the inverse cascade is absent. Consistently, we observed values of $\left\langle p^{3}\right\rangle \simeq 0$ (within statistical uncertainties). It would be interesting to investigate the correlation between the statistics of power and metenstrophy by means of simulations that resolve simultaneously the two cascades, changing independently their extension.

An attempt to unify the two measures of irreversibility could be made by introducing a combination of the energy and enstrophy, e.g., $\Psi=\int k^{a}\left[Z(k)+k_{f}^{2} E(k)\right]=\int k^{a}\left(k^{2}+\right.$ $\left.k_{f}^{2}\right) E(k)$, where $E(k)$ is the energy spectrum, $Z(k)=k^{2} E(k)$ is the enstrophy spectrum, and $0<a<2 / 3$. Assuming a Kraichnan spectrum $E(k) \sim k^{-5 / 3}$ for $k_{1}<k<k_{f}$ and $E(k) \sim k^{-3}$ for $k_{f}<k<k_{2}$, one has that the quantity $\Psi$ is dominated by the energy contribution in the low-wave-number range with a factor $\left(k_{f} / k_{1}\right)^{2 / 3-a}$ and by enstrophy in the high-wave-number range with a factor $\left(k_{2} / k_{f}\right)^{a}$. The statistics of time derivatives (or time increments) of $\Psi$ along Lagrangian trajectories could therefore provide a suitable global measure of the irreversibility of 2D turbulence, encompassing the phenomenology of the dual cascade.
[1] U. Frisch, Turbulence: The Legacy of A. N. Kolmogorov (Cambridge University Press, Cambridge, 1995).

[2] G. Falkovich and A. Frishman, Phys. Rev. Lett. 110, 214502 (2013).

[3] H. Xu, A. Pumir, G. Falkovich, E. Bodenschatz, M. Shats, H. Xia, N. Francois, and G. Boffetta, Proc. Natl. Acad. Sci. USA 111, 7558 (2014).

[4] A. Pumir, H. Xu, E. Bodenschatz, and R. Grauer, Phys. Rev. Lett. 116, 124502 (2016).

[5] G. Boffetta and R. E. Ecke, Annu. Rev. Fluid Mech. 44, 427 (2012).

[6] T. Grafke, A. Frishman, and G. Falkovich, Phys. Rev. E 91, 043022 (2015).

[7] A. Pumir, H. Xu, G. Boffetta, G. Falkovich, and E. Bodenschatz, Phys. Rev. X 4, 041006 (2014).

[8] R. H. Kraichnan, Phys. Fluids 10, 1417 (1967).

[9] G. Boffetta and S. Musacchio, Phys. Rev. E 82, 016307 (2010).
[10] M. E. Maltrud and G. K. Vallis, J. Fluid Mech. 228, 321 (1991).

[11] V. Borue, Phys. Rev. Lett. 71, 3967 (1993).

[12] T. Gotoh, Phys. Rev. E 57, 2984 (1998).

[13] A. Belmonte, W. Goldburg, H. Kellay, M. Rutgers, B. Martin, and X. Wu, Phys. Fluids 11, 1196 (1999).

[14] E. Lindborg and K. Alvelius, Phys. Fluids 12, 945 (2000).

[15] C. Pasquero and G. Falkovich, Phys. Rev. E 65, 056305 (2002).

[16] S. Chen, R. E. Ecke, G. L. Eyink, X. Wang, and Z. Xiao, Phys. Rev. Lett. 91, 214501 (2003).

[17] M. K. Rivera, W. B. Daniel, S. Y. Chen, and R. E. Ecke, Phys. Rev. Lett. 90, 104502 (2003).

[18] G. Boffetta, A. Celani, S. Musacchio, and M. Vergassola, Phys. Rev. E 66, 026304 (2002).

[19] A. Bracco and J. C. McWilliams, J. Fluid Mech. 646, 517 (2010). 